\title{
Objective and Subjective Measures of Visual Aesthetics of Website Interface Design: The Two Sides of the Coin
}

\author{
Ahamed Altaboli ${ }^{1,2}$ and Yingzi Lin ${ }^{1}$ \\ ${ }^{1}$ Mechanical and Industrial Engineering Department, \\ Northeastern University, 360 Huntington Avenue, Boston, MA 02115, USA \\ ${ }^{2}$ Industrial and Manufacturing Systems, Engineering Department, \\ University of Garyounis, Benghazi, Libya \\ Altaboli@gmail.com, yilin@coe.neu.edu
}

\begin{abstract}
The main purpose of this study is to compare objective layout-based measures of visual aesthetics with subjective questionnaire-based measures. Correlation analysis was used to carry out the comparison. Values for the tested objective measures were calculated for forty-two web pages already used in a previous study, for which subjective questionnaire scores (classical/expressive and VisA WI) were already available. Results showed significant correlations between many of the tested objective screen layout-based measures and subjective questionnaire-based measures related to order and layout of the screen. These findings suggest that the objective layout-based measures tested in this study can be used for overall assessments of visual aesthetics of websites and particularly for assessing aesthetic aspects related to the classical and the simplicity dimensions of website aesthetics.
\end{abstract}

Keywords: Aesthetics measures, Measure of Website aesthetics, Classical/expressive aesthetics, VisA WI, Visual aesthetics.

\section{Introduction}

Since its establishment in earlier 1980s, the field of the Human Computer Interaction (HCI) was mainly concerned with functionality and usability aspects of interactive computerized systems. However, recently, there was a new wave in the field emphasizing the importance of aesthetic aspects in HCI and interface design [13, 14, \& 21].

\subsection{Visual Aesthetics in Interface Design}

The attention to the importance of aesthetics in interface design and its effect on users' impressions of usability of the system began with findings of Kurosu and Kashimura study [9]. Using different designs of an automated teller machine interface, they found high correlation between users' prior perception of usability (they called it apparent usability) and users' perception of visual aesthetics of the interface. Participants perceived the visually appealing interface designs as easier to use. Later, Tracinsky [29] repeated their experiment in a different context using more rigors approach, same high correlation was also found in all the tested cases. Furthermore, 
this strong relationship between user perception of interface aesthetics and perceived usability remains intact even after actual use of the system [27]. Lindgaard et al. [12] showed that first impressions of perception of visual appeal of websites formed very quickly within 50 milliseconds. It remains stable even after a considerably longer exposure [27]. Phillips and Chapparro [23] found that users' impression of usability of websites is most influenced by the visual appeal of the site. Users rated sites with high visual appeal and low usability as easier to use, and gave lower rates to sites with low visual appeal and high usability.

Besides positive effect of aesthetics on perceived usability, some even argue that visually appealing interfaces might also have positive effects on performance. For example, Moshagen et al., [17] found significant effect of highly aesthetic websites on completion time in a low usability condition when participants completed search tasks. Sonderegger \& Sauer [26] showed that visual appearance of cell phones had a positive effect on performance, leading to reduced completion time and number of errors for the visually appealing design.

With recognition of the effect of visual aesthetics of interface and computer screen design on users' overall evaluation of the system, the next natural step in the design process is to develop tools to assess and measure visual aesthetics.

\subsection{Measures of Interface Aesthetics}

In general, two approaches to measure interface aesthetics can be distinguished in the literature. The first is an objective approach relating screen design features and layout elements to the users' perception of visual aesthetics (e.g. [3 \& 20]). The second one is a subjective approach, utilizing questionnaire-based instruments to measure users' perception of visual aesthetics (e.g. [11].

Objective Screen Layout-based Measures. This approach represents a bottom-up procedure. It has its roots in the rationalistic philosophical view of aesthetics [22]. This approach comprises the concept of "beauty in the observed object"; i.e. human perception of beauty is based on the order and organization of the various components constructing the object. It is concerned with determining what features in the interface design triggers users' precipitin of aesthetics of the interface. It also tries to explore the possibility of expressing changes in such features using numerical values and use these numerical values to assess users' perception of interface aesthetics. Methods in this approach are motivated by earlier aesthetic measures developed by Birkhoff [5], Tullis' quantitative techniques for evaluating screen design [32], and Gestlest theory for visual design [6 \& 19].

Supports of this approach [3 \& 19] argue that developing such measures can be very helpful in many design situations. They can be extremely helpful in early stages of design. They can assist in preparing design alternatives and can reduce the number of prototypes that will undergo tests with human users in later stages of design. However, they are not meant to be replacements to human designers, but are intended to serve as numerical tools to help designers and researches evaluate different design alternatives without the need to use human participants. Moreover, they can provide researchers with quantitative tools that can help in systematically study different design aspects and give a numerical basis for direct comparing of different design proposals. These 
measures can also be useful in cases where on-the-fly designs are needed for non professional designers as in online tools for designing websites [10].

Methods in this approach can be divided into two categories; one that simply uses numerical counts of visual features on the screen (like: number of objects, number of images ...etc) and relates them to users' perception of aesthetics. The second one uses mathematical formulas to express more sophisticated visual design features and concepts (like: symmetry, balance ...etc) and relate them to users' perception of aesthetics.

Simple counts measures. Visual features used in this categories includes number of constructing elements or blocks and chunks of information on the screen [3, 4, \& 15], number of images [ $3,4,7, \& 15]$, image size and font size [7 \& 25], JPEG file size of screenshots of websites [31]

Formularized measures. Methods in this category argues that physical layout of visual objects on the screen may play a role in users' perception of aesthetics. The procedure involves expressing visual design features (like symmetry, balance, unity ...etc) using mathematical formulas and combine calculated values for all features to build an overall measure that would reflect aesthetic level of the interface design.

One of such measures is the model developed by Ngo et al. [19]. The model consists of fourteen proposed measures of screen aesthetics: balance, symmetry, equilibrium, unity, sequence, density, proportions, cohesion, simplicity, regularity, economy, homogeneity, rhythm, and order. The value of each measure can be calculated using formulas based on the layout of visual objects on the screen. The average of all these measures represents the overall aesthetic value of the screen.

In a practical application of the model, Zain et al. [33] designed a computer application to incorporate five measures (balance, equilibrium, symmetry, sequence, and rhythm) of the fourteen measures. They used the software to evaluate language learning web pages.

Bauerly and Liu [3 \& 4] developed mathematical formulas to measure and test effects of symmetry and balance on interface aesthetics. They developed their formulas based on a microscopic view that compare screen elements in question pixel by pixel, as opposed to the macroscopic view used by Ngo et al. [20] that compare higher level elements such as specific objects or shapes. However, similar results were obtained in both studies, which suggest that there are no practical differences between the two sets of formulas. Lai et al. [10] utilized the quantitative measures of symmetry and balance used by Bauerly and Liu [3 \& 4] to quantitatively analyze the aesthetics of a text-overlaid image such that a best position for overlaying the texts on a background image can be obtained automatically. The two measures were evaluated against participants' subjective rating of visual aesthetic appeal in cases of color and monochrome images.

Subjective Questionnaire-based Measures. Supporters of this approach claim that the complexity and interrelated relationships among the screen design elements make it difficult to use them to quantitatively measure aesthetics [11]. It would be more convenient to use questionnaire-based instruments to measure users' subjective perception of aesthetics. Two widely accepted of such instruments are: the classical and expressive instrument developed by Lavie and Tractinsky [11], and the Visual 
Aesthetics of Website Inventory (VisAWI) tool developed by Moshagen and Thielsch [16]. Both were designed to measure perceived visual aesthetics of websites.

Lavie and Tractinsky [11] found two dimensions of the perceived website aesthetics, termed "classical aesthetics" and "expressive aesthetics". The classical aesthetics dimension emphasizes orderly and clear design and is closely related to many of the usability and interface design rules and guidelines. The expressive aesthetics dimension is linked to the designers' creativity and originality and to the ability to break design conventions. These two dimensions were the basis for developing quantitative questionnaire-based instrument to measure website interface aesthetics. The classical dimension includes the items "aesthetic", "pleasant", "symmetric", "clear", and "clean", while the expressive aesthetics includes the items "creative", "fascinating", "original", "sophisticated", and "uses special effects".

VisAWI was constructed to serve as a new tool to measure perceived website aesthetics. It was designed to provide a tool that would cover border aspects of perceived websites aesthetics that weren't adequately presented in early instruments. The instrument is based on four interrelated facets of perceived visual aesthetics of websites: simplicity, diversity, colorfulness, and craftsmanship. Simplicity comprises visual aesthetics aspects such as balance, unity, and clarity. It is closely related to the classical aesthetics dimension. The Diversity facet comprises visual complexity, dynamics, novelty, and creativity. It is closely related to the expressive aesthetics dimension. The colorfulness facet represents aesthetic impressions perceived from the selection, placement, and combination of colors. Craftsmanship comprises the skillful and coherent integration of all relevant design dimensions. Each of the first two facets is presented by five items in the questionnaire, while each of the last two facets has four items. For full list of items of both questionnaires see [11 \& 16].

\subsection{Purpose of the Study}

The main concern of this study is to investigate the possibility of finding significant correlations between objective and subjective measures of website aesthetics. The hypothesis is that significant correlations could be -at least- found between the objective layout-based measures and items related to screen layout in the subjective questionnaire-based measures (classical measure in the classical/expressive questionnaire and simplicity measure in the VisA WI questionnaire).

Forty-two web pages already used in a previous study [16] to develop and validate the VisA WI questionnaire and compare it with classical and expressive aesthetics questionnaire will be used in this study. Values of selected objective measures will be calculated for these 42 web pages and compared to subjective questionnaire scores (VisA WI and classical/expressive) already available in [16]. Correlation analysis will be used to carry out the comparisons.

The reason why these 42 web pages are utilized in this study is that they cover a wide variety of websites with different levels of visual aesthetics. In addition, questionnaire scores (for both VisA WI and classical/expressive tools) for a relatively large sample size are already available for these pages. 


\section{Results and Discussion}

\subsection{Selected Objective Measures for the Study}

Formularized. Eight of the measures suggested by Ngo et al. [18] were selected for this study. The reasons for selecting these measures are that they are widely accepted and have already being used and tested in many previous studies [1, 18, 24, \& 33]. The eight selected measures are: symmetry, balance, unity, sequence, simplicity, density, economy, and rhythm. The formulas developed by Ngo et al. [18] will be used to calculate values for the selected measures (for full details of how to calculate these measures and their definitions see $[18,19, \& 20])$. Table 1 shows descriptive statistics for the calculated values using the formalized selected measures for the 42 web pages. Possible range of values of each measure is zero to one, where zero represents the lowest aesthetic level and one represents the highest level.

Table 1. Descriptive statistics for the selected formularized measures for the 42 web pages

\begin{tabular}{lcccc}
\hline Measure & Min & Max & Average & Standard deviation \\
\hline Symmetry & 0.761 & 0.985 & 0.873 & 0.046 \\
Balance & 0.516 & 0.950 & 0.792 & 0.105 \\
Unity & 0.157 & 0.684 & 0.393 & 0.145 \\
Sequence & 0.750 & 1.000 & 0.970 & 0.082 \\
Simplicity & 0.077 & 0.273 & 0.152 & 0.044 \\
Density & 0.091 & 0.977 & 0.453 & 0.230 \\
Economy & 0.050 & 0.250 & 0.105 & 0.040 \\
Rhythm & 0.257 & 0.792 & 0.627 & 0.109 \\
\hline
\end{tabular}

Counts. Five measures were selected in this category, namely: number of visual objects on the screen, number of different sizes of visual objects, number of images, number of different font types used in the web page, and JEPG file size of screenshot of the webpage. Number of objects, number of images, and JEPG files size have already been tested in previous studies [2, 3, 16, 14 \& 24]; all with results indicting some sort of a relationship between these measures and users' perception of visual aesthetics. Number of different sizes of visual objects is one of the input parameters in $\mathrm{Ngo}$ et al. formulas for unity, economy, and simplicity; authors were interested in testing it as a single feature. Number of different font types has been selected based on earlier observation. Descriptive statistics for the calculated values for the selected count-based measures for the 42 web pages are given in Table 2 .

The calculated values for the above selected measures for all the 42 web pages will be compared with scores of the classical/expressive and VisA WI questionnaires (see [16] for questionnaire scores given to the 42 web pages). 
Table 2. Descriptive statistics for the selected count-based measures for the 42 web pages

\begin{tabular}{lcccc}
\hline Measure & Min & Max & Average & Standard deviation \\
\hline No of objects & 6 & 22 & 11.9 & 3.9 \\
No of different sizes of objects & 4 & 20 & 10.7 & 3.6 \\
JEPG file size* & 50 & 251 & 170.8 & 44.4 \\
No of different font types & 1 & 6 & 2.8 & 1.3 \\
No of images & 0 & 12 & 4.3 & 3.1 \\
& & & &
\end{tabular}

* in Kbytes

\subsection{Correlation Analysis}

Correlation analysis was used to investigate possible relationship between the selected objective and subjective measures of visual aesthetics of websites. Values of all the selected objective measures were calculated for the 42 web pages, scores of subjective questionnaire-based measures were obtained from [16]

Table 3 shows correlation coefficients between the formalized measures and questionnaire scores for the 42 web pages. From the table, one can see that all significant correlations are with questionnaire items related to screen layout. The objective measures of unity, simplicity, and economy are significantly correlated with the classical and the simplicity measures; both containing items related to visual layout and clarity of the design. No significant correlations were found between the formalized measures and questionnaire scores related to the expressive aesthetics.

An unexpected result is the lack of significant correlations between symmetry and balance, and any of the questionnaire scores. This contradicts with results of earlier studies showing significant effects of symmetry and balance on visual interface aesthetics [1, 3, 4, 10, 18, \& 30]. Possible explanation could be found by looking at findings of an earlier study conducted by authors of the current study [2]. The study investigated effects of the measures of balance, unity, and sequence and their interactions on perceived visual aesthetics. Findings showed that with each pair of measures the effect of one measure is larger at high values of the other measure; with the low values the effect is very small. Looking back at Table 3, it can be seen that values of symmetry, balance, and sequence are much higher than values of measures with significant correlations (unity, simplicity, and economy). Symmetry, balance, and sequence have values larger than $0.761,0.516$, and 0.750 respectively with averages of $0.873,0.792$, and 0.970 respectively. On the other hand, measures with significant correlations have smaller values; Unity with values less than 0.684 and an average of 0.393 , simplicity and economy with values less than 0.300 and averages of 0.152 and 0.105 respectively.

Since symmetry, balance, and sequence have higher values than unity, simplicity and economy, therefore, the later three will have higher effects on perceived aesthetics. This might explain why only these three measures have significant correlations. Nevertheless, the other case of lower values of symmetry, balance, and sequence should also be investigated to confirm this explanation. Also, Can the high levels of 
symmetry and balance witnessed in the current study be considered as a typical characteristic of all websites designs? Or is it just a coincidence with the 42 web pages used in the study?

Another point worth mentioning is that only measures that have number of different sizes of objects as an input parameter in their formulas (unity, simplicity, and economy) produced significant correlations. The measures of density and rhythm that do not have this parameter in their formulas didn't produce significant correlations, though have low values.

Table 4 shows correlation coefficients between the simple counts-based measures and questionnaire scores for the 42 web pages. Significant correlations were found between number of objects and number of different sizes with both the classical and the simplicity measures. This wasn't surprising, since these two features (no of objects and no of different sizes) are the main input parameters in the formulas used to calculate values of the formalized-based measures that showed significant correlations (unity, simplicity, and economy; for full details of the used formulas see [18, 19, \& 20]). These significant correlations point out to clear negative effects of increasing number of objects and number of different sizes on perceived visual aesthetics of websites. The questions now are: Is there an optimal value or a threshold for these two features? How much can both of them be lowered? Do they affect each other?

No strong correlations were found between JEPG file size, number of different font types, and number of images with any of the classical and the simplicity measures. However, an interesting result is the noticeable high and significant correlations found between number of different fonts and the expressive and the diversity measures. Does this indicate that number of different fonts may affect visual aesthetics? More examinations are needed to clarify this.

\section{Conclusions}

The main goal of this study was to test the possibility of that significant correlations could be found between the objective layout-based measures of website visual aesthetics and the subjective questionnaire-based measures related to order and organization of visual objects on the screen. Values for the selected objective measure were calculated for forty-two web pages already used in a previous study [16] for which subjective questionnaire scores (classical/expressive and VisA WI) were already available. Correlation coefficients between the objective measures and questionnaire scores were calculated. Results showed significant correlations between many of the selected objective screen layout-based measures and the subjective questionnairebased measures related to order and layout of the screen.

This suggests that the objective layout-based measures tested in this study could be used to generally assess the overall visual aesthetics of websites and particularly aesthetic aspects related to classical and simplicity dimensions of website aesthetics.

However, several issues still need to be considered when interpreting findings of this study. First, the formulas used to calculate values of the objective layout-based measures don't include effect of colors, although, Ngo et al [17] suggested adding effects of colors as part of the balance measure, but, it is still not clear how to express 

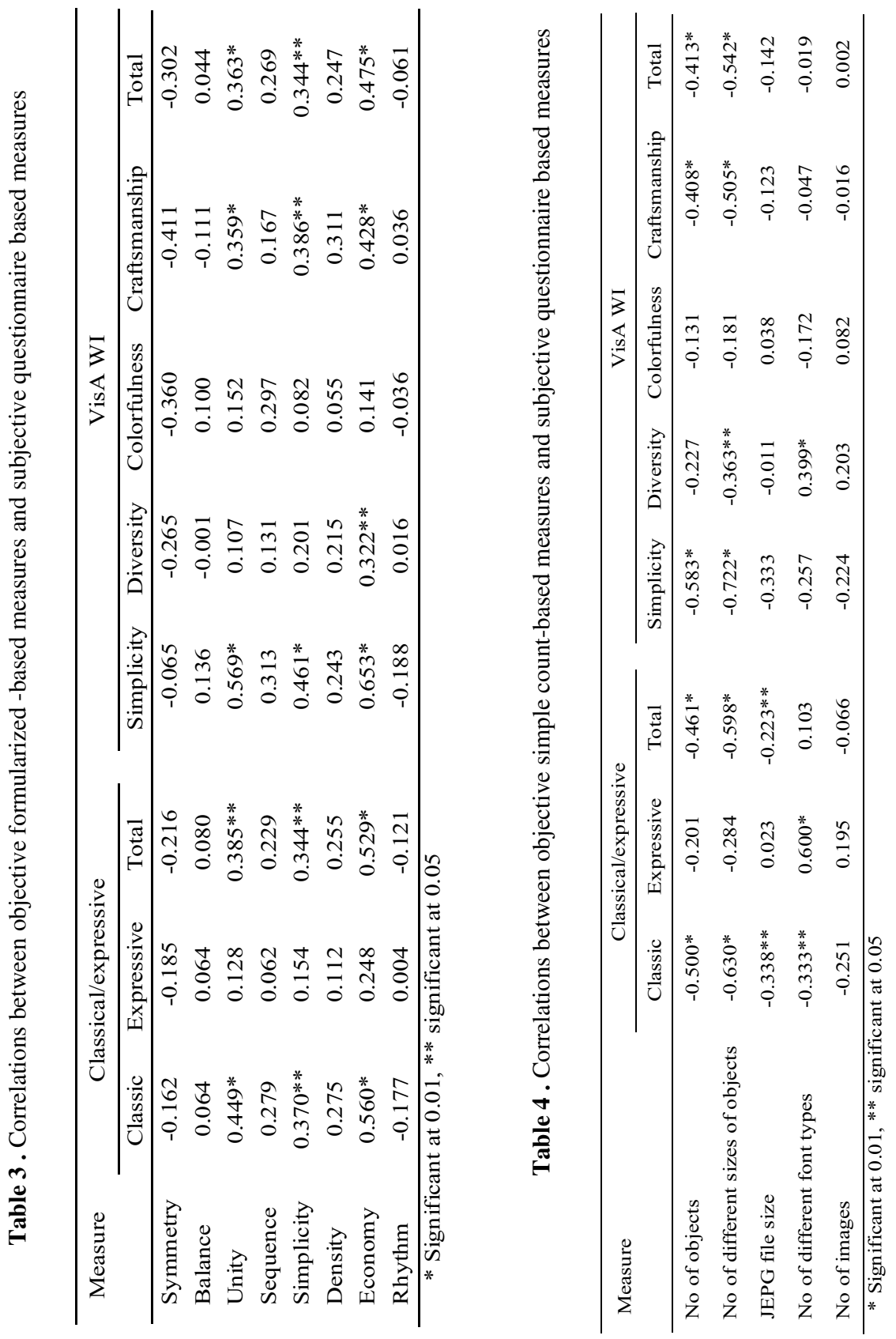
effects of colors using numerical values. Second, for many of the relationships suggested by the significant correlations found in this study to be fully confirmed, further testing using more rigorous procedures is needed. Finally, since web pages were used in this study, findings of this study are only applicable to visual aesthetics of websites. Nonetheless, it would be interesting to see how these findings would work with other types of interfaces and screens (e.g. cell phones).

Acknowledgements. The authors would like to thank Morten Moshagen and Meinald T.Thielsch (authors of reference [16]) for providing the screenshots and questionnaire scores for the 42 web pages. This research has been supported by NSF through a CAREER program (Award \# 0954579).

\section{References}

1. Altaboli, A., Lin, Y.: Experimental Investigation of Effects of Balance, Unity, and Sequence on Interface and Screen Design Aesthetics. In: Blashki, K. (ed.) Proceedings of The IADIS International Conference Interface and Human Computer Interaction 2010, Freiburg, Germany, pp. 243-250. IADIS Press (2010)

2. Altaboli, A., Lin, Y.: Investigating the Effects of Screen Layout Elements on Interface and Screen Design Aesthetics. Technical Report (unpublished). Intelligent Human-Machine Systems Lab, Mechanical and Industrial Engineering Department, Northeastern University, Boston, MA, USA (2010)

3. Bauerly, M., Liu, Y.: Computational modeling and experimental investigation of effects of compositional elements on interface and design aesthetics. Int. J. Human-Computer Studies 64, 670-682 (2006)

4. Bauerly, M., Liu, Y.: Effects of Symmetry and Number of Compositional Elements on Interface and Design Aesthetics. International Journal of Human- Computer Interaction 24(3), 275-287 (2008)

5. Birkhoff, G.: Aesthetic Measure. Harvard University Press, Cambridge (1933)

6. Chand, D., Dooley, L., Tuovinen, E.: Gestalt Theory in Visual Screen Design - A New Look at an Old Subject. Presented at the Seventh World Conference on Computer in Education, Copenhagen, Denmark. Australian Computer Society, Inc. (2002)

7. Djamasbia, S., Siegelb, M., Tullisb, T.: Generation Y, web design, and eye tracking. Int. J. Human-Computer Studies 68, 307-323 (2010)

8. Hartmann, J., Sutcliffe, A., De Angeli, A.: Towards a theory of user judgment of aesthetics and user interface quality. ACM Transactions on Computer-Human Interaction 15(4), 1-30 (2008)

9. Kurosu, M. and Kashimura, K.: Apparent usability vs. inherent usability: experimental analysis on the determinants of the apparent usability. In: CHI 1995: Conference Companion on Human Factors in Computing Systems, Denver, Colorado, United States, pp. 292293 (1995)

10. Lai, C., Chen, P., Shih, S., Liu, Y., Hong, J.: Computational models and experimental investigations of effects of balance and symmetry on the aesthetics of text-overlaid images. Int. J. Human-Computer Studies 68, 41-56 (2010)

11. Laviea, T., Tractinsky, N.: Assessing dimensions of perceived visual aesthetics of web sites. Int. J. Human-Computer Studies 60, 269-298 (2004) 
12. Lindgaard, G., Fernandez, G., Dudek, C., Brown, J.: Attention web designers: You have 50 milliseconds to make a good impression. Behavior \& Information Technology 25(2), 115126 (2006)

13. Liu, Y.: Engineering aesthetics and aesthetic ergonomics: theoretical foundations and a dual-process research methodology. Ergonomics 46, 1273-1292 (2003)

14. Liu, Y.: The aesthetic and the ethic dimensions of human factors and design. Ergonomics 46, 1293-1305 (2003)

15. Michailidou, E., Harper, S., Bechhofer, S.: Visual Complexity and Aesthetic Perception of Web pages. In: SIGDOC 2008, Lisbon, Portugal, September 22-24 (2008)

16. Moshagen, M., Thielsch, M.T.: Facets of visual aesthetics. International Journal of Human-Computer Studies 68(10) (2010)

17. Moshagen, M., Musch, J., Göritz, A.S.: A blessing, not a curse: Experimental evidence for beneficial effects of visual aesthetics on performance. Ergonomics 52, 1311-1320 (2009)

18. Ngo, D., Byrne, J.: Application of an aesthetic evaluation model to data entry screens. Computers in Human Behavior 17, 149-185 (2001)

19. Ngo, D.C.L., Teo, L.S., Byrne, J.G.: Evaluating Interface Esthetics. Knowledge and Information Systems (4), 46-79 (2002)

20. Ngo, D.C.L., Teo, L.S., Byrne, J.G.: Modelling interface aesthetics. Information Sciences 152(1), 25-46 (2003)

21. Norman, D.: Emotional design: Why we love (or hate) everyday things. Basic Books, New York (2004)

22. Reich, Y.: A model of aesthetic judgment in design. Artificial Intelligence in Engineering 8(2), 141-153 (1993)

23. Phillips, C., Chapparro, C.: Visual Appeal vs. Usability: Which One Influences User Perceptions of a Website More? Usability News 11(2) (2009)

24. Salimun, C., Purchase, H.C., Simmons, D.R., Brewster, S.: Preference ranking of screen layout principles. In: The 24th BCSHCI 2010, Abertay Dundee, September 6-10 (2010)

25. Schmidt, K.E., Bauerly, M., Liu, Y., Sridharan, S.: Web Page Aesthetics and Performance: A Survey and an Experimental Study. In: Proceedings of the 8th Annual International Conference on Industrial Engineering - Theory, Applications and Practice, Las Vegas, Nevada, USA (2003)

26. Sonderegger, A., Sauer, J.: The influence of design aesthetics in usability testing: Effects on user performance and perceived usability. Applied Ergonomics 41, 403-410 (2010)

27. Tractinsky, N., Cokhavi, A., Kirschenbaum, M., Sharfi, T.: Evaluating the consistency of immediate aesthetic perceptions of web pages. International Journal of Human-Computer Studies 64(11), 1071-1083 (2006)

28. Tractinsky, N., Shoval-Katz, A., Ikar, D.: What is beautiful is usable. Interacting with Computers 13, 127-145 (200)

29. Tractinsky, N.: Aesthetics and apparent usability: empirically assessing cultural and methodological issues. In: Pemberton, S. (ed.) Proceedings of the 1997 Conference on Human Factors in Computing Systems (CHI 1997). ACM Press, New York (1997)

30. Tuch, A., Bargas-Avila, J., Opwis, K.: Symmetry and Aesthetics in Website Design: It's a man's Business. Computers in Human Behavior 26, 1831-1837 (2010)

31. Tuch, A.N., Bargas-Avila, J.A., Opwis, K., Wilhelm, F.H.: Visual complexity of websites: Effects on users' experience, physiology, performance, and memory. International Journal of Human-Computer Studies 67, 703-715 (2009)

32. Tullis, T.S.: The formatting of alphanumeric displays: a review and analysis. Human Factors 25(6), 657-682 (1983)

33. Zain, J., Tey, M., Goh, Y.: Probing a Self-Developed Aesthetics Measurement Application (SDA) in Measuring Aesthetics of Mandarin Learning Web Page Interfaces. IJCSNS International Journal of Computer Science and Network Security 8(1) (2008) 